\title{
Glycerol Uptake Mutants of the Hyphal Fungus Aspergillus nidulans
}

\author{
By JAAP VISSER, ${ }^{*}$ RUTGER VAN ROOIJEN, ${ }^{1}$ COR DIJKEMA, ${ }^{2}$ \\ KLAAS SWART ${ }^{1}$ AND HEATHER M. SEALY-LEWIS ${ }^{3}$ \\ ${ }^{1}$ Department of Genetics and ${ }^{2}$ Department of Molecular Physics, Agricultural University, \\ Generaal Foulkesweg 53, 6703 BM Wageningen, The Netherlands \\ ${ }^{3}$ Department of Biochemistry, University of Hull, Hull HU6 $7 R X, U K$
}

(Received 19 October 1987)

\begin{abstract}
A new class of glycerol non-utilizing mutants, designated $g l c C$, has been isolated. The $g l c C$ gene was mapped in linkage group VI and mutants were found to complement the reference strains $g l c A I$ (linkage group V) and $g l c B 33$ (linkage group I) in diploids. The new mutants were unable to grow on glycerol. However, in contrast to the $g l c A$ and $g l c B$ phenotype these mutants did grow well on dihydroxyacetone and D-galacturonate. By in vivo ${ }^{13} \mathrm{C}$ NMR spectroscopy it was shown that the $g l c C$ mutant did not take up glycerol but did take up dihydroxyacetone. The latter substrate was converted intracellularly into glycerol which was then catabolized as normal.
\end{abstract}

\section{INTRODUCTION}

Glycerol metabolism in filamentous fungi has thus far been studied most extensively in Neurospora crassa. Evidence was presented for a catabolic pathway involving phosphorylation to glycerol 3-phosphate and subsequent oxidation of glycerol 3-phosphate in the mitochondria to dihydroxyacetone phosphate (Courtright, 1975). This route was indeed confirmed as glycerol non-utilizing mutants were isolated that lacked either glycerol kinase $(\mathrm{glpl})$ or the mitochondrial membrane-bound flavoprotein glycerol-3-phosphate dehydrogenase ( $\mathrm{glp} 2$ ) (Holm et al., 1976; Denor \& Courtright, 1978, 1982). However, Viswanath-Reddy et al. (1977) and Tom et al. (1978) have also suggested that there is an alternative route in which glycerol is utilized by a direct $\mathrm{NADP}^{+}$-dependent oxidation to glyceraldehyde which is similar to the $\mathrm{NAD}^{+}$-dependent oxidation to dihydroxyacetone in Schizosaccharomyces pombe and in some other yeasts (Marshall et al., 1985).

In Aspergillus nidulans Payton (1978) isolated two classes of glycerol non-utilizing mutants, $g l c A$ and $g l c B$, after mutation and filtration enrichment in a glycerol medium. Filtration enrichment in a D-galacturonate medium, however, resulted only in mutants of the glcB genotype (Uitzetter et al., 1986). We have recently found biochemical evidence that in $A$. nidulans glycerol is also catabolized through phosphorylation $(g l c A)$ and oxidation $(g l c B)(\mathrm{J}$. Visser \& coworkers, unpublished results). In this fungus the presence of a NADP ${ }^{+}$-dependent glycerol dehydrogenase was also established. The latter enzyme is involved in the conversion of dihydroxyacetone into glycerol. The interaction between D-galacturonate breakdown and glycerol catabolism was also previously shown (Uitzetter et al., 1986). The uronic acid is catabolized through glyceraldehyde and pyruvate, the former compound being further metabilized through glycerol. In this study we describe the isolation and characterization of a new class of glycerol non-utilizing mutants $(\mathrm{glcC})$ in addition to the two genotypes already described.

\section{METHODS}

Strains and mutagenesis. The strains of Aspergillus were all derived from the original Glasgow strains (Pontecorvo et al., 1953). The strain used for mutagenesis was $\mathrm{H} 542$ (pabaAl al X4 sB43) from which the glycerol non-utilizing strain $\mathrm{H} 913$ was derived. The wild-type control was WG096 (pabaA1 yA2). The glycerol non- 


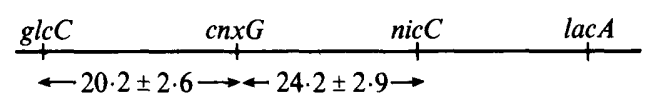

Fig. 1. Map of linkage group VI. Map distances \pm 1 SD are expressed in centiMorgans. For the map distance nicC to lacA see Clutterbuck (1984).

utilizing strains WG196 (pabaAl yA2 glcAl) and WG197 (pabaAl yA2glcB33) were used in growth and complementation tests. $y A 2$ pantoB100 was used to outcross the mutagenized glycerol-containing strains to produce relevant genotypes for mitotic haploidization and complementation tests. Master strain, MSD, was used for assigning the $g l c^{-}$mutations to a linkage group and the markers $c n x G 4$, nicCl and lacAl were used for mapping the position of $g l c C$. The markers were described by Clutterbuck (1984). The genetic techniques have been modified after Pontecorvo et al. (1953), McCully \& Forbes (1965) and Clutterbuck (1974).

Media and growth tests. WG096 and H913 were grown on complete medium (CM) according to Pontecorvo et al. (1953) to obtain conidia for submerged cultures. Growth tests were done on minimal medium (MM) supplemented with $p$-aminobenzoate $\left(2 \mathrm{mg} \mathrm{l}^{-1}\right)$ and DL-methionine $\left(100 \mathrm{mg} \mathrm{l}^{-1}\right)$.

Carbon sources were added separately as membrane filter sterilized solutions to a final concentration of $0.05 \mathrm{M}$ of each individual carbon source. The growth of the wild-type and mutant strains was scored after $2 \mathrm{~d}$ incubation at $37^{\circ} \mathrm{C}$. Submerged cultures for ${ }^{13} \mathrm{C}$ NMR spectroscopy were obtained by inoculating conidiospores $\left(10^{6} \mathrm{ml}^{-1}\right)$ in minimal medium using D-glucose as carbon source and supplemented with $p$-aminobenzoate and DL-methionine. Mycelia were grown for $18 \mathrm{~h}$ in a Gallenkamp orbital shaker using $300 \mathrm{ml}$ of medium in 1 litre flasks. Transfer of mycelium to media containing glycerol or dihydroxyacetone was done as described before (Dijkema et al., 1985).

${ }^{13} \mathrm{C} N M R$ spectroscopy. The ${ }^{13} \mathrm{C}$ NMR spectra were obtained at $75.46 \mathrm{MHz}$ on a Bruker CXP-300 NMR spectrometer operating in the Fourier transform mode and equipped with a $10 \mathrm{~mm}$ dedicated ${ }^{13} \mathrm{C}$ probe. Details of sample preparation and measuring conditions have been published before (Dijkema et al., 1985).

\section{RESULTS AND DISCUSSION \\ Identification and mapping of glcC}

The strain pabaAl al X4 sB43 was mutagenized with 4-nitroquinoline-1-oxide (Bal et al., 1977) and the conidia were plated on medium appropriate for the selection of alcA/alcR mutants ( $50 \mathrm{mM}$-glycerol as carbon source with the addition of $2.5 \mathrm{~mm}$-allyl alcohol). Strains that lack alcohol dehydrogenase I (ADHI) either because of a defect in the structural gene, alcA, or a defect in the positively acting regulatory gene, alcR, are unable to metabolize allyl alcohol to its 'toxic' product, acrolein, and are therefore able to grow. A large number of allyl alcohol resistant mutants were obtained and the genetic characterization of mutations in alc $A$ and alcR will be described in detail elsewhere (H. M. Sealy-Lewis, unpublished data), but some properties of these mutations have already been published (Sealy-Lewis \& Lockington, 1984). Among a selection of 50 allyl alcohol resistant mutants examined in detail there were two strains carrying alcR mutations, alcR5217 and alcR5233, that were unable to grow on glycerol medium ( $50 \mathrm{~mm}$ glycerol as carbon source). The strains were outcrossed to $y A 2$ pantoB 100 and it was found that the inability to grow on glycerol medium segregated independently from the alcR mutation. Complementation tests were done in diploids between the two $\mathrm{glc}^{-}$mutants, which failed to complement, but showed growth similar to a wild-type control diploid on glycerol medium when tested with either $g l c A 1$ or $g l c B 33$. The new mutations were designated $g l c C l$ (derived from the alcR5233 strain) and $g l c C 2$ (derived from the alcR5217 strain). In a cross between a strain carrying $g l c C l$ and $g l c C 2$ there were no progeny that were able to grow on glycerol medium (40 progeny tested). A mitotic haploidization analysis involving a glc $C$ strain and MSD with all the linkage groups marked indicated that the $g l c C$ mutation segregated with linkage group VI. Further mapping studies have positioned $g l c C$ on the left arm of linkage group VI (see Fig. 1). A four point cross involving glcC, $c n x G$, nicC and lac $A$ gives the order of markers $g l c C, c n x G$, nicC and lacA. nic $C$ and lacA have been previously mapped (see Clutterbuck, 1984) and this makes glcC the most extreme marker on the left arm of linkage group VI.

\section{Phenotypic characterization of $\mathrm{glcC}$}

The growth properties of the $g l c C$ mutants were compared with those of the two other known glycerol non-utilizing mutant classes $g l c A$ and $g l c B$. The results are summarized in Table 1. 


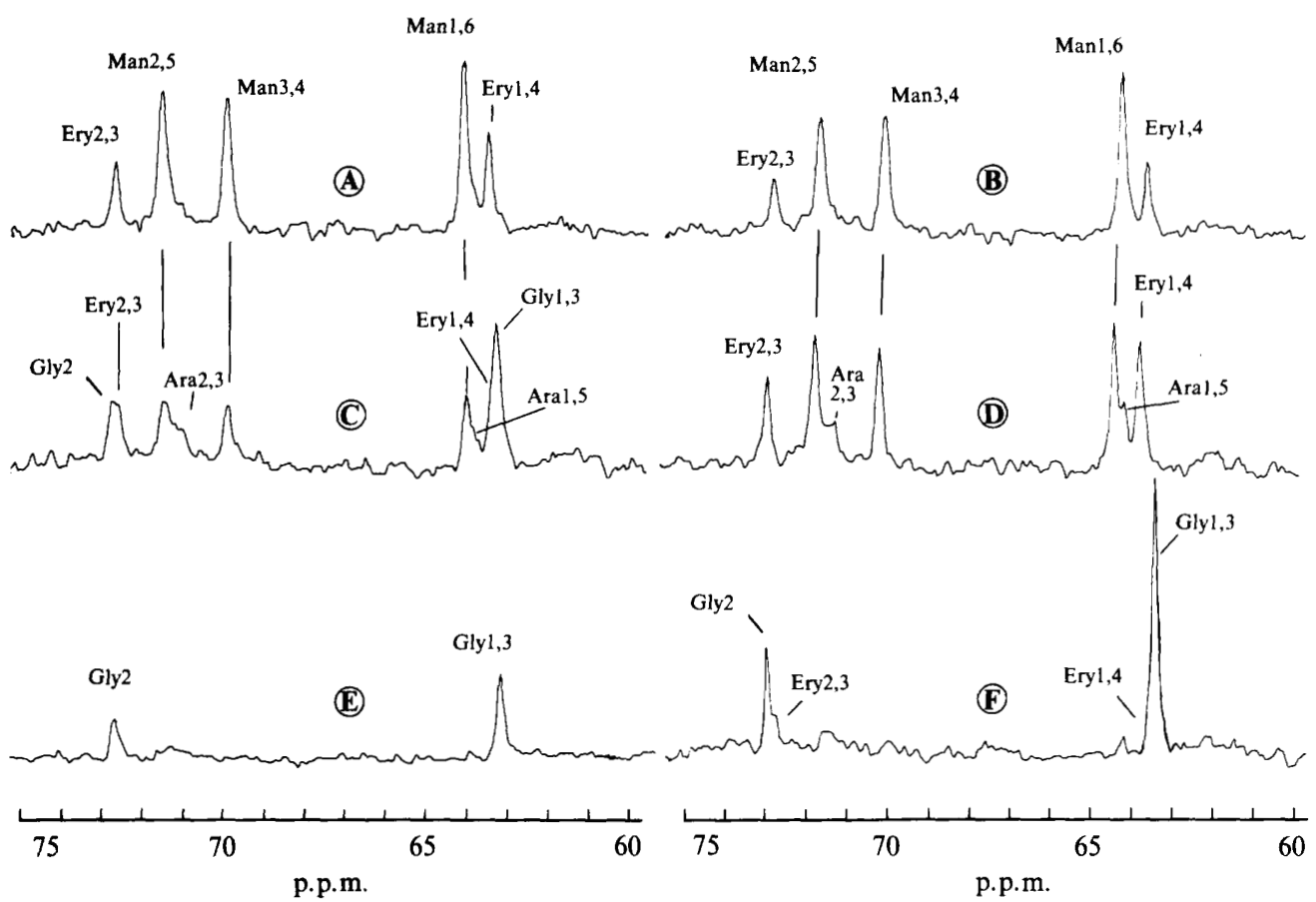

Fig. 2. Natural abundance ${ }^{13} \mathrm{C}$ NMR spectra of the polyol resonance region of wild-type $A$. nidulans WG096 (spectra A, C and E) and of the glcC mutant strain H913 (spectra B, D and F). A, B, spectra taken after $18 \mathrm{~h}$ of growth on D-glucose $(0.1 \mathrm{M}) ; \mathrm{C}, \mathrm{D}$, spectra taken after a $2 \mathrm{~h}$ transfer period to glycerol $(0 \cdot 1 \mathrm{M}) ; \mathrm{E}, \mathrm{F}$, spectra taken after a $2 \mathrm{~h}$ transfer period to dihydroxyacetone $(0 \cdot 1 \mathrm{M})$.

Table 1. Growth characteristics of $A$. nidulans glcA, B and $C$ mutants ++++ , Good growth $;++$, moderate; + , very poor; - , no growth.

Growth of strain:

\begin{tabular}{lllll}
\multicolumn{1}{c}{ Carbon source } & WG096 & glcA & glcB & glcC \\
D-Glucose & ++++ & ++++ & ++++ & ++++ \\
Glycerol & +++ & + & - & - \\
Dihydroxyacetone & +++ & - & - & +++ \\
D-Galacturonate & +++ & + & - & +++ \\
D-Glucose/glycerol & ++++ & +++ & - & ++++
\end{tabular}

Whereas $g l c A$ and $g l c B$ mutants were unable to utilize dihydroxyacetone, D-galacturonate and glycerol, glc $C$ mutants did grow well on the former two substrates. In $A$. nidulans dihydroxyacetone is converted into glycerol by a NADP ${ }^{+}$-dependent glycerol dehydrogenase activity as indicated previously. Moreover, D-galacturonate and glycerol catabolism have at least one step in common (Uitzetter et al., 1986). Since glcC mutants grow well on dihydroxyacetone and D-galacturonate this suggests a defect in a very early step of glycerol catabolism in these new mutants. They were suspected to be defective in their glycerol uptake.

${ }^{13} \mathrm{C} N M R$ spectroscopy of $A$. nidulans wild-type and glcC mutant strains

In order to verify whether glycerol uptake was indeed impaired in the glcC mutant $\mathrm{H} 913$, ${ }^{13} \mathrm{C}$ NMR spectra of wild-type and of mutant mycelium obtained under different incubation conditions were compared. Mycelia of both strains, grown on glucose for $18 \mathrm{~h}$, were found to have identical natural abundance ${ }^{13} \mathrm{C} N \mathrm{NR}$ spectra (Fig. 2, A and B). Resonances characteristic for the presence of mannitol and erythritol were found in both strains in similar 
intensities. After growth on glucose, mycelium was then transferred to a minimal medium containing either glycerol or dihydroxyacetone. After an incubation period of $2 \mathrm{~h}$ the spectra of both strains were again compared. In the case of a transfer to glycerol the wild-type spectrum and the $g l c C$ spectrum were found to be different (Fig. 2, C and D). In the wild-type the spectral changes are (i) the appearance of glycerol, characterized by resonances at 63.5 p.p.m. (C-1 and C-3) and at $73 \cdot 2$ p.p.m. (C-2), which differ in intensity and (ii) the decrease in intensity of both the mannitol and erythritol resonances. The C-2 and C-3 resonances of erythritol at 73.0 p.p.m. only appear as a shoulder on the resonance at 73.2 p.p.m. of glycerol, which clearly accumulates intracellularly. In the mutant spectrum, however, hardly any spectral change occurred in the $2 \mathrm{~h}$ time span (cf. Fig. 2, spectrum B), and in the natural abundance spectra glycerol resonances do not show up.

Upon transfer to a minimal medium containing dihydroxyacetone only glycerol resonances are found in the natural abundance ${ }^{13} \mathrm{C}$ NMR spectra both in the wild-type and the mutant (Fig. $2, \mathrm{E}$ and $\mathrm{F}$ ). Thus uptake of dihydroxyacetone by the fungus is immediately followed by its reduction to glycerol, which requires NADPH and the presence of a constitutive glycerol dehydrogenase. The other polyol pools have disappeared and thus seem to accommodate the cellular requirement for NADPH by increased catabolism.

Starvation of the mycelium even for prolonged periods of time ( $6 \mathrm{~h}$ or more) does not deplete the polyol pools to the same extent as observed upon incubation with dihydroxyacetone. These results explain why dihydroxyacetone itself is never observed in the mycelial spectra after its uptake. A rapid conversion of this toxic compound into an inert metabolite (glycerol) is required. Since dihydroxyacetone can also arise intracellularly from dihydroxyacetone 3phosphate this explains the constitutive nature of glycerol dehydrogenase at high levels.

In conclusion our ${ }^{13} \mathrm{C}$ NMR spectra of this $g l c C$ mutant show that, when glycerol is given externally, it does not appear in the mycelium. However, when glycerol is generated intracellularly from dihydroxyacetone it can serve as a carbon source leading to normal growth behaviour. These results identify $g l c C$ as a glycerol uptake mutant. We have subsequently tested $g l c C$ for growth on glycerol over a wide range of glycerol concentrations $(50 \mathrm{mM}-1 \mathrm{M})$. No growth at all was found for the mutant even at a $1 \mathrm{~m}$ concentration. It thus seems that even at high concentrations diffusion of glycerol is insufficient for growth and that there is an absolute requirement for a specific glycerol transport system.

This study is part of the Agricultural University Biotechnology Programme on Biocatalysts.

\section{REFERENCES}

Bal, J., Kajtaniak, E. M. \& Pieniazek, N. J. (1977). 4-Nitroquinoline-1-oxide: a good mutagen for Aspergillus nidulans. Mutation Research 56, 153-156.

CLUTTERBUCK, A. J. (1974). In Handbook of Genetics, pp. 447-510. Edited by R. C. King. New York: Plenum Press.

ClutTERBUCK, A. J. (1984). Loci and linkage map of the filamentous fungus Aspergillus nidulans (Eidam) Winter $(n=8)$. Genetic Maps 3, 265-273.

COURTRIGHT, J. B. (1975). Intracellular localization and properties of glycerokinase and glycerophosphate dehydrogenase in Neurospora crassa. Archives of Biochemistry and Biophysics 167, 21-33.

DENOR, P. F. \& COURTRIGHT, J. B. (1978). Isolation and characterization of glycerol-3-phosphate dehydrogenase-defective mutants of Neurospora crassa. Journal of Bacteriology 136, 960-968.

DENOR, P. F. \& CoURTRIGHT, J. B. (1982). Genetic and enzymatic characterization of the inducible glycerol dissimilatory system of Neurospora crassa. Journal of Bacteriology 151, 912-917.
Dijkema, C., Kester, H. C. M. \& Visser, J. (1985). ${ }^{13}$ C NMR studies of carbon metabolism in the hyphal fungus Aspergillus nidulans. Proceedings of the $\mathrm{Na}$ tional Academy of Sciences of the United States of America 82, 14-18.

Holm, K., Nilheden, E., Kolmark, H. G. \& Burton, K. (1976). Genetic and enzymatic analysis of a glycerol kinase deficient mutant in Neurospora crassa. Molecular and General Genetics 144, 11-15.

Marshall, J. H., May, J. W. \& Sloan, J. (1985). Purification and properties of glycerol: $\mathrm{NAD}^{+} 2$ oxidoreductase (glycerol dehydrogenase) from Schizosaccharomyces pombe. Journal of General Microbiology 131, 1581-1588.

MCCully, K. S. \& Forbes, E. (1965). The use of $p$ fluorophenylalanine with master strains of Aspergillus nidulans for assigning genes to linkage groups. Genetical Research 6, 352-359.

PAYTon, M. A. (1978). A genetic study of sugar metabolism and transport in Aspergillus nidulans. $\mathrm{PhD}$ thesis, University of Leicester, UK. 
Pontecorvo, G., Roper, J. A., Hemmons, L. J., MCDonald, K. D. \& Bufton, A. W. J. (1953). The genetics of Aspergillus nidulans. Advances in Genetics 5, 141-238.

SEALy-Lewis, H. M. \& Lockington, R. A. (1984). Regulation of two alcohol dehydrogenases in Aspergillus nidulans. Current Genetics 8, 253-259.

Tom, G. D., Viswanath-Reddy, M. \& Howe, H. B., $J_{R}$ (1978). Effect of carbon source on enzymes involved in glycerol metabolism in Neurospora crassa. Archives of Microbiology 117, 259-263.
Uitzetter, J. H. A. A., Bos, C. J. \& Visser, J. (1986). Characterization of Aspergillus nidulans mutants in carbon metabolism isolated after D-galacturonate enrichment. Journal of General Microbiology 32, 1167-1172.

VISWANATH-REDDY, M., BENNETT, S. N. \& Howe, H. B. (1977). Characterization of glycerol nonutilizing and protoperithecial mutants of Neurospora. Molecular and General Genetics 153, 29-38. 